\title{
Integrated Weed Management in Chilli + Garlic Intercropping System
}

\author{
Vilas D. Gasti* and Snehasish Chakravorty \\ Department of Horticulture and Post-harvest Technology PalliSikshaBhavana (Institute of \\ Agriculture) Sriniketan, Visva-Bharati (West Bengal) - 731236, India
}

*Corresponding author

\begin{abstract}
A B S T R A C T
Keywords

Weed management, Chilli, Garlic, Intercropping system

Article Info

Accepted:

26 December 2018

Available Online:

10 January 2019

Intercropping system plays an important role in increasing land use efficiency and weed suppression. A field experiment entitled "Integrated weed management in chilli + garlic intercropping system" was conducted at the Vegetable Division in Kittur Rani Channamma College of Horticulture, Arabhavi, University of Horticultural Sciences, Bagalkot (Karnataka) during kharif 2014 and 2015. The objective of the experiment was to identify the best possible method of weed control for maximizing the productivity of chilli+garlic intercropping system. Among the different chemical treatments, pre-emergent application of alachlor @ $1.5 \mathrm{~kg}$ ai $/ \mathrm{ha}+2 \mathrm{HW}$ at 45 and 60 DAT recorded the lowest pooled dry matter of weeds $(2.59 \mathrm{~g})$, lowest monocot (3.57), dicot weeds (3.06) and weed population (4.7) thus exhibited the highest weed control efficiency $(87.85 \%)$. The yield per ha of green chilli (256.93 q) and garlic (30.80 q), net returns (Rs.1,08,565) and B:C ratio (2.30) were found to be highest and it was followed by treatment with pre-emergent application of pendimethalin at $1.5 \mathrm{~kg} / \mathrm{ha}+2 \mathrm{HW}$ at 45 and $60 \mathrm{DAT}$.
\end{abstract}

\section{Introduction}

Intercropping is a primitive practice and it has been recognized as useful as it results in a greater crop canopy which may ensure maximum utilization of resources and in addition provides an environment unfavourable for weed growth. Intercropping of chilli with different vegetables offer greater scope to utilize the land and other resources to the maximum extent. Chillit garlic intercropping system is one of the most assured intercropping system and suppress the weeds to some extent and increases the yield and is found suitable to northern dry zone of Karnataka.
India has been known as the "Home of Spices" from very ancient time. Both chilli and garlic are used as spice and condiment and are widely used for seasoning and flavouring food Apart from vegetables. Garlic is closely planted between the chilli and shallow rooted bulbous crop. Therefore, intercultural practices are very difficult to undertake and manual weeding during the establishment stage of crop causes physical damage to the crop plants. A most troublesome problem faced by growers is the control of weed particularly during the early stage of crop growth and thus crop suffer heavily from weed competition. The weeds compete for the nutrients, moisture, space and 
light and affect growth and development. Weed reduces the yield to the extent of 40-80 per cent and therefore, it is essential to keep the field weed free during the critical period of crop growth (Mohite et al., 2015). Therefore, the aim of the study is to identify the best potential weed control treatment suitable for chilli+ garlic intercropping system.

\section{Materials and Methods}

The study was carried out at the Vegetable Division in Kittur Rani Channamma College of Horticulture, Arabhavi, University of Horticultural Sciences, Bagalkot (Karnataka.) during kharif season of 2014 and 2015 on well drained red loamy soil to find out the effectiveness of chemicals in weed management in intercropping of chilli (Var 'Byadagi') + garlic (Local Variety). The gross size and net size of the plots were $16.20 \mathrm{~m}^{2}$ and $11.80 \mathrm{~m}^{2}$ respectively. Four to five weeks old chilli seedlings were transplanted into main field with a spacing of $75 \mathrm{~cm} \times 45 \mathrm{~cm}$ and in between the chilli, 2 rows of garlic was planted.

The field experiment was laid out in Randomized Complete Block Design (RCBD) with two replications consisting of 14 treatments including unweeded check $\left(\mathrm{T}_{1}\right.$. Alachlor (PE)-1.5 kg ai/haT 2 - Alachlor (PE)$1.0 \mathrm{~kg}$ ai $/ \mathrm{haT}_{3}$ - Alachlor (PE)-1.5 kg ai $/ \mathrm{ha}+1$ $\mathrm{HW}$ at $45 \mathrm{DATT}_{4}$ - Alachlor (PE)-1.5 kg ai/ha $+2 \mathrm{HW}$ at 45 and $60 \mathrm{DATT}_{5}$ - Alachlor (PE)$1.0 \mathrm{~kg}$ ai/ha $+1 \mathrm{HW}$ at $45 \mathrm{DATT}_{6}$ - Alachlor (PE)-1.0 kg ai/ha $+2 \mathrm{HW}$ at 45 and 60 DATT $_{7}$ _Pendimethalin (PE)-1.5 kg ai/ $/ \mathrm{haT}_{8}{ }^{-}$ Pendimethalin (PE)-1.0 kg ai/haT . $_{9}$. Pendimethalin (PE)-1.5 kg ai/ha+ $1 \mathrm{HW}$ at 45 DATT $_{10}$ - Pendimethalin (PE)- $1.5 \mathrm{~kg}$ ai $/ \mathrm{ha}+2$ $\mathrm{HW}$ at 45 and $60 \mathrm{DATT}_{11}$ - Pendimethalin (PE)-1.0 kg ai/ha $+1 \mathrm{HW}$ at $45 \mathrm{DATT}_{12}$ Pendimethalin (PE)-1.0 kg ai $/ \mathrm{ha}+2 \mathrm{HW}$ at 45 and 60 DATT $_{13}$ - Weed free checkT 14 . Unweeded check).RDF for chilli,
150:100:125 kg of $\mathrm{N}: \mathrm{P}_{2} \mathrm{O}_{5}: \mathrm{K}_{2} \mathrm{O}$ with 25 tonnes of FYM (as per package of practice UHS, Bagalkot) was applied at the time of field preparation. Remaining dose of N (50 $\%$ ) was applied at 3 split doses viz., after 30, 60 and 90 days of transplanting. During the course of investigation, observations regarding weed population, crop growth parameters and yield parameters at 30, 60, 90 DAT and at the time of harvest were recorded from the randomly selected and tagged plants. The weed index was calculated by the formula given by Gill and Vijayakumar (1969). Besides fixed cost of cultivation, variable costs on spray, manual weeding and cost of herbicide in each treatment was worked out to obtain total cost of production. The net income was obtained after deducting cost of production from value of produces. The mean data was subjected to the statistical analysis using ANOVA and mean separation (LSD) procedures (Gomez and Gomez, 1984).

\section{Results and Discussion}

The results of the study showed that among different chemical treatments, the effect of weed control on monocot weeds(3.57), dicot weeds (3.06) and weed population at harvest (4.70) was found to be significantly less in treatment $\mathrm{T}_{4}$ (Alachlor (PE)-1.5 kg ai/ha + $2 \mathrm{HW}$ at 45 and 60 DAT). But it was found to be on par with $\mathrm{T}_{10}$ (Pendimethalin (PE)-1.5 kg ai/ha $+2 \mathrm{HW}$ at 45 and 60 DAT) (Table 1). The treatment $\mathrm{T}_{4}$ recorded lowest dry weight of weeds at harvest $(2.59 \mathrm{~g})$, lowest weed index in chilli and garlic (16.25 and 2.08, respectively) and thus highest weed control efficiency (87.85\%) (Table -2). The lower dry weight of weeds in these treatments might be attributed to the less number of weeds. Thus the higher weed control efficiency could be accounted to the lower weed dry weight. These results are of agreement with Ningappa (2013), Shil and Adhikary (2014) and Chaudhari et al., (2017). 
Table.1 Effect of weed control treatments on weed parameters in chilli + garlic intercropping

\begin{tabular}{|c|c|c|c|c|c|c|c|c|c|c|}
\hline \multirow[t]{2}{*}{ Treatments } & \multirow[t]{2}{*}{ Treatment details } & \multicolumn{3}{|c|}{ Monocot weeds at harvest } & \multicolumn{3}{|c|}{ Dicot weeds at harvest } & \multicolumn{3}{|c|}{ Weed population at harvest } \\
\hline & & $\begin{array}{c}\text { I } \\
\text { year }\end{array}$ & $\begin{array}{c}\text { II } \\
\text { year }\end{array}$ & Pooled & $\begin{array}{c}\text { I } \\
\text { year }\end{array}$ & $\begin{array}{c}\text { II } \\
\text { year }\end{array}$ & Pooled & $\begin{array}{c}\text { I } \\
\text { year }\end{array}$ & $\begin{array}{c}\text { II } \\
\text { year }\end{array}$ & Pooled \\
\hline $\mathbf{T}_{1}$ & Alachlor (PE)-1.5 kg ai/ha & $\begin{array}{l}53.80 \\
(7.33)\end{array}$ & $\begin{array}{l}57.67 \\
(7.59)\end{array}$ & $\begin{array}{l}55.74 \\
(7.47)\end{array}$ & $\begin{array}{l}27.89 \\
(5.28)\end{array}$ & $\begin{array}{l}27.29 \\
(5.22)\end{array}$ & $\begin{array}{l}27.59 \\
(5.25)\end{array}$ & $\begin{array}{l}81.69 \\
(9.04)\end{array}$ & $\begin{array}{l}84.96 \\
(9.22)\end{array}$ & $\begin{array}{l}83.32 \\
(9.13)\end{array}$ \\
\hline $\mathbf{T}_{2}$ & Alachlor (PE)-1.0 kg ai/ha & $\begin{array}{l}62.90 \\
(7.93)\end{array}$ & $\begin{array}{l}64.50 \\
(8.03)\end{array}$ & $\begin{array}{l}63.70 \\
(7.98)\end{array}$ & $\begin{array}{l}36.68 \\
(6.06)\end{array}$ & $\begin{array}{l}36.29 \\
(6.02)\end{array}$ & $\begin{array}{l}36.48 \\
(6.04)\end{array}$ & $\begin{array}{l}99.58 \\
(9.98)\end{array}$ & $\begin{array}{l}100.79 \\
(10.04)\end{array}$ & $\begin{array}{l}100.18 \\
(10.01)\end{array}$ \\
\hline $\mathbf{T}_{3}$ & Alachlor (PE)-1.5 kg ai/ha $+1 \mathrm{HW}$ at $45 \mathrm{DAT}$ & $\begin{array}{l}37.10 \\
(6.09)\end{array}$ & $\begin{array}{l}37.16 \\
(6.09)\end{array}$ & $\begin{array}{l}37.13 \\
(6.09)\end{array}$ & $\begin{array}{l}17.44 \\
(4.17)\end{array}$ & $\begin{array}{l}16.19 \\
(4.02)\end{array}$ & $\begin{array}{l}16.81 \\
(4.10)\end{array}$ & $\begin{array}{l}54.54 \\
(7.38)\end{array}$ & $\begin{array}{l}53.34 \\
(7.30)\end{array}$ & $\begin{array}{l}53.94 \\
(7.34)\end{array}$ \\
\hline $\mathbf{T}_{4}$ & Alachlor (PE)-1.5 kg ai/ha $+2 \mathrm{HW}$ at 45 and $60 \mathrm{DAT}$ & $\begin{array}{l}12.43 \\
(3.52)\end{array}$ & $\begin{array}{l}13.15 \\
(3.62)\end{array}$ & $\begin{array}{l}12.79 \\
(3.57)\end{array}$ & $\begin{array}{c}9.87 \\
(3.14)\end{array}$ & $\begin{array}{c}8.84 \\
(2.97)\end{array}$ & $\begin{array}{c}9.35 \\
(3.06)\end{array}$ & $\begin{array}{l}22.30 \\
(4.72)\end{array}$ & $\begin{array}{l}21.99 \\
(4.69)\end{array}$ & $\begin{array}{l}22.14 \\
(4.70)\end{array}$ \\
\hline $\mathbf{T}_{5}$ & Alachlor (PE)-1.0 kg ai/ha $+1 \mathrm{HW}$ at $45 \mathrm{DAT}$ & $\begin{array}{l}42.00 \\
(6.48)\end{array}$ & $\begin{array}{l}42.36 \\
(6.51)\end{array}$ & $\begin{array}{l}42.18 \\
(6.49)\end{array}$ & $\begin{array}{l}18.92 \\
(4.35)\end{array}$ & $\begin{array}{l}18.39 \\
(4.28) \\
\end{array}$ & $\begin{array}{l}18.65 \\
(4.32)\end{array}$ & $\begin{array}{l}60.92 \\
(7.80) \\
\end{array}$ & $\begin{array}{l}60.74 \\
(7.79)\end{array}$ & $\begin{array}{l}60.83 \\
(7.80)\end{array}$ \\
\hline $\mathbf{T}_{6}$ & Alachlor (PE)-1.0 kg ai/ha $+2 \mathrm{HW}$ at 45 and $60 \mathrm{DAT}$ & $\begin{array}{l}28.63 \\
(5.35)\end{array}$ & $\begin{array}{l}30.16 \\
(5.49)\end{array}$ & $\begin{array}{l}29.40 \\
(5.42)\end{array}$ & $\begin{array}{l}11.43 \\
(3.38)\end{array}$ & $\begin{array}{l}11.84 \\
(3.44)\end{array}$ & $\begin{array}{l}11.63 \\
(3.41)\end{array}$ & $\begin{array}{l}38.97 \\
(6.24) \\
\end{array}$ & $\begin{array}{l}40.25 \\
(6.34)\end{array}$ & $\begin{array}{l}39.61 \\
(6.29)\end{array}$ \\
\hline $\mathbf{T}_{7}$ & Pendimethalin (PE)-1.5 kg ai/ha & $\begin{array}{l}60.60 \\
(7.78)\end{array}$ & $\begin{array}{l}62.18 \\
(7.88)\end{array}$ & $\begin{array}{l}61.39 \\
(7.83)\end{array}$ & $\begin{array}{l}33.68 \\
(5.80)\end{array}$ & $\begin{array}{l}32.39 \\
(5.69)\end{array}$ & $\begin{array}{l}33.03 \\
(5.75)\end{array}$ & $\begin{array}{l}94.28 \\
(9.71)\end{array}$ & $\begin{array}{l}94.57 \\
(9.72)\end{array}$ & $\begin{array}{l}94.42 \\
(9.72)\end{array}$ \\
\hline $\mathbf{T}_{8}$ & Pendimethalin (PE)-1.0 kg ai/ha & $\begin{array}{l}66.80 \\
(8.17)\end{array}$ & $\begin{array}{l}70.28 \\
(8.38)\end{array}$ & $\begin{array}{l}68.54 \\
(8.28)\end{array}$ & $\begin{array}{l}40.22 \\
(6.34)\end{array}$ & $\begin{array}{l}36.79 \\
(6.06)\end{array}$ & $\begin{array}{l}38.50 \\
(6.20)\end{array}$ & $\begin{array}{l}107.02 \\
(10.34)\end{array}$ & $\begin{array}{l}107.06 \\
(10.35)\end{array}$ & $\begin{array}{l}107.04 \\
(10.34)\end{array}$ \\
\hline $\mathbf{T}_{9}$ & Pendimethalin (PE)-1.5 kg ai/ha+ $1 \mathrm{HW}$ at $45 \mathrm{DAT}$ & $\begin{array}{l}45.03 \\
(6.71)\end{array}$ & $\begin{array}{l}45.10 \\
(6.71)\end{array}$ & $\begin{array}{l}45.06 \\
(6.71)\end{array}$ & $\begin{array}{l}21.43 \\
(4.62)\end{array}$ & $\begin{array}{l}20.29 \\
(4.50)\end{array}$ & $\begin{array}{l}20.86 \\
(4.57)\end{array}$ & $\begin{array}{l}66.46 \\
(8.15)\end{array}$ & $\begin{array}{l}65.38 \\
(8.09)\end{array}$ & $\begin{array}{l}65.92 \\
(8.12)\end{array}$ \\
\hline $\mathbf{T}_{10}$ & Pendimethalin (PE)-1.5 kg ai/ha $+2 \mathrm{HW}$ at 45 and $60 \mathrm{DAT}$ & $\begin{array}{l}14.45 \\
(3.80)\end{array}$ & $\begin{array}{l}17.09 \\
(4.12)\end{array}$ & $\begin{array}{l}15.77 \\
(3.97)\end{array}$ & $\begin{array}{l}10.34 \\
(3.21)\end{array}$ & $\begin{array}{l}10.09 \\
(3.17)\end{array}$ & $\begin{array}{l}10.21 \\
(3.20)\end{array}$ & $\begin{array}{l}25.88 \\
(5.09)\end{array}$ & $\begin{array}{l}28.92 \\
(5.38)\end{array}$ & $\begin{array}{l}27.40 \\
(5.23)\end{array}$ \\
\hline $\mathbf{T}_{11}$ & Pendimethalin (PE)-1.0 kg ai/ha $+1 \mathrm{HW}$ at $45 \mathrm{DAT}$ & $\begin{array}{l}53.23 \\
(7.30)\end{array}$ & $\begin{array}{l}54.08 \\
(7.35)\end{array}$ & $\begin{array}{l}53.66 \\
(7.32)\end{array}$ & $\begin{array}{l}25.52 \\
(5.05)\end{array}$ & $\begin{array}{l}23.39 \\
(4.83)\end{array}$ & $\begin{array}{l}24.45 \\
(4.94)\end{array}$ & $\begin{array}{l}78.75 \\
(8.87)\end{array}$ & $\begin{array}{l}77.47 \\
(8.80)\end{array}$ & $\begin{array}{l}78.11 \\
(8.84)\end{array}$ \\
\hline $\mathbf{T}_{12}$ & Pendimethalin (PE)-1.0 kg ai/ha $+2 \mathrm{HW}$ at 45 and $60 \mathrm{DAT}$ & $\begin{array}{l}31.10 \\
(5.58)\end{array}$ & $\begin{array}{l}31.60 \\
(5.62)\end{array}$ & $\begin{array}{l}31.35 \\
(5.60)\end{array}$ & $\begin{array}{l}11.99 \\
(3.46)\end{array}$ & $\begin{array}{l}14.89 \\
(3.85)\end{array}$ & $\begin{array}{l}13.44 \\
(3.66)\end{array}$ & $\begin{array}{l}43.09 \\
(6.56)\end{array}$ & $\begin{array}{l}46.49 \\
(6.82)\end{array}$ & $\begin{array}{l}44.79 \\
(6.69)\end{array}$ \\
\hline $\mathbf{T}_{13}$ & Weed free check & $\begin{array}{c}0.00 \\
(0.71)\end{array}$ & $\begin{array}{c}00.00 \\
(0.71)\end{array}$ & $\begin{array}{c}0.00 \\
(0.71)\end{array}$ & $\begin{array}{c}0.00 \\
(0.71)\end{array}$ & $\begin{array}{c}0.00 \\
(0.71)\end{array}$ & $\begin{array}{c}0.00 \\
(0.71)\end{array}$ & $\begin{array}{c}0.00 \\
(0.71)\end{array}$ & $\begin{array}{c}0.00 \\
(0.71)\end{array}$ & $\begin{array}{c}0.00 \\
(0.71)\end{array}$ \\
\hline $\mathbf{T}_{14}$ & Unweeded check & $\begin{array}{c}105.48 \\
(10.27)\end{array}$ & $\begin{array}{c}112.54 \\
(10.61)\end{array}$ & $\begin{array}{c}109.01 \\
(10.44)\end{array}$ & $\begin{array}{l}65.44 \\
(8.09) \\
\end{array}$ & $\begin{array}{l}64.39 \\
(8.02) \\
\end{array}$ & $\begin{array}{l}64.91 \\
(8.06) \\
\end{array}$ & $\begin{array}{c}170.92 \\
(13.07)\end{array}$ & $\begin{array}{l}176.92 \\
(13.30)\end{array}$ & $\begin{array}{l}173.92 \\
(13.19)\end{array}$ \\
\hline & Mean & $\begin{array}{l}43.86 \\
(6.22)\end{array}$ & $\begin{array}{l}45.60 \\
(6.34)\end{array}$ & $\begin{array}{l}44.73 \\
(6.28)\end{array}$ & $\begin{array}{l}23.67 \\
(4.55)\end{array}$ & $\begin{array}{l}22.96 \\
(4.48)\end{array}$ & $\begin{array}{l}23.32 \\
(4.52)\end{array}$ & $\begin{array}{l}67.46 \\
(7.64)\end{array}$ & $\begin{array}{l}68.56 \\
(7.77)\end{array}$ & $\begin{array}{l}67.97 \\
(7.67)\end{array}$ \\
\hline & S. Em \pm & 0.08 & 0.15 & 0.09 & 0.12 & 0.15 & 0.08 & 0.10 & 0.07 & 0.07 \\
\hline & C.D. @ 5\% & 0.25 & 0.46 & 0.27 & 0.37 & 0.45 & 0.25 & 0.23 & 0.21 & 0.21 \\
\hline
\end{tabular}


Table.2 Effect of weed control treatments on weed parameters in chilli + garlic intercropping

\begin{tabular}{|c|c|c|c|c|c|c|c|c|c|c|c|c|c|}
\hline \multirow[t]{2}{*}{ Treaments } & \multirow[t]{2}{*}{ Treatment details } & \multicolumn{3}{|c|}{$\begin{array}{c}\text { Dry weight of weeds at } \\
\text { harvest }(g)\end{array}$} & \multicolumn{3}{|c|}{$\begin{array}{l}\text { Weed control efficiency } \\
\text { at harvest }\end{array}$} & \multicolumn{3}{|c|}{ Weed index in chilli } & \multicolumn{3}{|c|}{ Weed index in garlic } \\
\hline & & $\begin{array}{c}\text { I } \\
\text { year }\end{array}$ & $\begin{array}{c}\text { II } \\
\text { year }\end{array}$ & Pooled & $\begin{array}{c}\text { I } \\
\text { year }\end{array}$ & $\begin{array}{c}\text { II } \\
\text { year }\end{array}$ & Pooled & $\begin{array}{c}\text { I } \\
\text { year }\end{array}$ & $\begin{array}{c}\text { II } \\
\text { year }\end{array}$ & Pooled & $\begin{array}{c}\text { I } \\
\text { year }\end{array}$ & $\begin{array}{c}\text { II } \\
\text { year }\end{array}$ & Pooled \\
\hline $\mathbf{T}_{1}$ & Alachlor (PE)-1.5 kg ai/ha & $\begin{array}{l}31.69 \\
(5.63)\end{array}$ & $\begin{array}{l}35.95 \\
(5.99)\end{array}$ & $\begin{array}{l}33.82 \\
(5.81)\end{array}$ & 42.36 & 35.19 & 38.77 & 20.89 & 24.55 & 22.62 & 24.29 & 31.11 & 27.70 \\
\hline $\mathbf{T}_{2}$ & Alachlor (PE)-1.0 kg ai/ha & $\begin{array}{l}34.27 \\
(5.85)\end{array}$ & $\begin{array}{l}45.58 \\
(6.74)\end{array}$ & $\begin{array}{l}39.93 \\
(6.32)\end{array}$ & 37.72 & 17.86 & 27.79 & 24.25 & 26.47 & 25.20 & 32.09 & 37.74 & 34.91 \\
\hline $\mathbf{T}_{\mathbf{3}}$ & $\begin{array}{l}\text { Alachlor (PE)-1.5 kg ai/ha }+1 \mathrm{HW} \text { at } 45 \\
\text { DAT }\end{array}$ & $\begin{array}{l}22.76 \\
(4.77)\end{array}$ & $\begin{array}{l}15.11 \\
(3.88)\end{array}$ & $\begin{array}{l}18.93 \\
(4.35)\end{array}$ & 58.60 & 72.84 & 65.72 & 17.62 & 23.66 & 20.86 & 10.66 & 17.88 & 14.27 \\
\hline $\mathbf{T}_{4}$ & $\begin{array}{l}\text { Alachlor (PE)-1.5 kg ai/ha }+2 \mathrm{HW} \text { at } 45 \\
\text { and } 60 \mathrm{DAT}\end{array}$ & $\begin{array}{c}3.48 \\
(1.86)\end{array}$ & $\begin{array}{l}10.01 \\
(3.16)\end{array}$ & $\begin{array}{c}6.74 \\
(2.59)\end{array}$ & 93.70 & 82.00 & 87.85 & 15.00 & 16.78 & 16.25 & 2.06 & 2.10 & 2.08 \\
\hline $\mathbf{T}_{5}$ & $\begin{array}{l}\text { Alachlor (PE)-1.0 kg ai/ha }+1 \mathrm{HW} \text { at } 45 \\
\text { DAT }\end{array}$ & $\begin{array}{l}24.89 \\
(4.99)\end{array}$ & $\begin{array}{l}19.90 \\
(4.45)\end{array}$ & $\begin{array}{l}22.39 \\
(4.73)\end{array}$ & 54.72 & 64.21 & 59.46 & 18.95 & 24.27 & 21.25 & 12.30 & 19.03 & 15.66 \\
\hline $\mathbf{T}_{6}$ & $\begin{array}{l}\text { Alachlor (PE)-1.0 kg ai/ha }+2 \mathrm{HW} \text { at } 45 \\
\text { and } 60 \text { DAT }\end{array}$ & $\begin{array}{l}11.26 \\
(3.35)\end{array}$ & $\begin{array}{l}12.57 \\
(3.53)\end{array}$ & $\begin{array}{l}11.91 \\
(3.45)\end{array}$ & 79.59 & 77.41 & 78.50 & 16.90 & 21.08 & 18.28 & 5.55 & 12.07 & 8.81 \\
\hline $\mathbf{T}_{7}$ & Pendimethalin (PE)-1.5 kg ai/ha & $\begin{array}{l}33.98 \\
(5.83)\end{array}$ & $\begin{array}{l}40.55 \\
(6.36)\end{array}$ & $\begin{array}{l}37.26 \\
(6.10)\end{array}$ & 38.19 & 26.88 & 32.54 & 22.81 & 25.47 & 23.11 & 28.29 & 34.90 & 31.59 \\
\hline $\mathbf{T}_{8}$ & Pendimethalin (PE)-1.0 kg ai/ha & $\begin{array}{l}37.09 \\
(6.09)\end{array}$ & $\begin{array}{l}49.74 \\
(7.05)\end{array}$ & $\begin{array}{l}43.41 \\
(6.59)\end{array}$ & 32.61 & 10.32 & 21.47 & 26.91 & 32.03 & 29.35 & 36.09 & 41.88 & 38.99 \\
\hline $\mathbf{T}_{9}$ & $\begin{array}{l}\text { Pendimethalin }(\mathrm{PE})-1.5 \mathrm{~kg} \text { ai/ha+ } 1 \mathrm{HW} \\
\text { at } 45 \mathrm{DAT}\end{array}$ & $\begin{array}{l}28.33 \\
(5.32)\end{array}$ & $\begin{array}{l}26.16 \\
(5.11)\end{array}$ & $\begin{array}{l}27.24 \\
(5.22)\end{array}$ & 48.46 & 52.88 & 50.67 & 19.62 & 22.53 & 22.12 & 17.93 & 24.79 & 21.36 \\
\hline $\mathbf{T}_{10}$ & $\begin{array}{l}\text { Pendimethalin }(\mathrm{PE})-1.5 \mathrm{~kg} \text { ai/ha }+2 \mathrm{HW} \\
\text { at } 45 \text { and } 60 \mathrm{DAT}\end{array}$ & $\begin{array}{l}4.15 \\
(2.03)\end{array}$ & $\begin{array}{l}11.47 \\
(3.38)\end{array}$ & $\begin{array}{c}7.81 \\
(2.79)\end{array}$ & 92.47 & 79.33 & 85.90 & 15.30 & 17.58 & 17.12 & 4.01 & 10.46 & 7.24 \\
\hline $\mathbf{T}_{11}$ & $\begin{array}{l}\text { Pendimethalin }(\mathrm{PE})-1.0 \mathrm{~kg} \text { ai } / \mathrm{ha}+1 \mathrm{HW} \\
\text { at } 45 \mathrm{DAT}\end{array}$ & $\begin{array}{l}28.56 \\
(5.34)\end{array}$ & $\begin{array}{l}31.12 \\
(5.57)\end{array}$ & $\begin{array}{l}29.84 \\
(5.46)\end{array}$ & 48.10 & 43.99 & 46.05 & 19.84 & 26.54 & 22.15 & 20.49 & 27.53 & 24.01 \\
\hline $\mathbf{T}_{12}$ & $\begin{array}{l}\text { Pendimethalin (PE)-1.0 kg ai/ha }+2 \mathrm{HW} \\
\text { at } 45 \text { and } 60 \mathrm{DAT}\end{array}$ & $\begin{array}{l}14.03 \\
(3.74)\end{array}$ & $\begin{array}{r}13.90 \\
(3.71)\end{array}$ & $\begin{array}{l}13.96 \\
(3.73)\end{array}$ & 74.48 & 75.04 & 74.76 & 17.53 & 21.22 & 20.17 & 8.62 & 16.18 & 12.40 \\
\hline $\mathbf{T}_{13}$ & Weed free check & $\begin{array}{c}0.00 \\
(0.71)\end{array}$ & $\begin{array}{c}0.00 \\
(0.71)\end{array}$ & $\begin{array}{c}0.00 \\
(0.71)\end{array}$ & 100.00 & 100.00 & 100.00 & 0.00 & 0.00 & 0.00 & 0.00 & 0.00 & 0.00 \\
\hline $\mathbf{T}_{14}$ & Unweeded check & $\begin{array}{l}55.02 \\
(7.42)\end{array}$ & $\begin{array}{l}55.39 \\
(7.44)\end{array}$ & $\begin{array}{l}55.21 \\
(7.43)\end{array}$ & 0.00 & 0.00 & 0.00 & 67.28 & 63.62 & 65.97 & 53.16 & 60.10 & 56.63 \\
\hline & Mean & $\begin{array}{l}23.57 \\
(4.50)\end{array}$ & $\begin{array}{l}26.28 \\
(4.79)\end{array}$ & $\begin{array}{l}24.92 \\
(4.66)\end{array}$ & $\mathbf{5 7 . 2 2}$ & 52.71 & 54.96 & 21.99 & 25.17 & 23.55 & 18.25 & 23.98 & 21.12 \\
\hline & S. Em \pm & 0.11 & 0.10 & 0.07 & 1.73 & 1.49 & 1.21 & 3.53 & 3.24 & 2.67 & 3.82 & 3.64 & 3.39 \\
\hline & C.D. @ 5\% & 0.32 & 0.29 & 0.21 & 5.29 & 4.55 & 3.70 & 10.76 & 9.91 & 7.77 & 11.65 & 11.12 & 10.34 \\
\hline
\end{tabular}


Table.3 Effect of weed control treatments on growth and yield parameters in chilli

\begin{tabular}{|c|c|c|c|c|c|c|c|c|c|c|c|c|c|}
\hline \multirow[t]{2}{*}{ Treatments } & \multirow[t]{2}{*}{ Treatment details } & \multicolumn{3}{|c|}{ Plant height at harvest $(\mathrm{cm})$} & \multicolumn{3}{|c|}{$\begin{array}{l}\text { No. of branches per } \\
\text { plant at harvest }\end{array}$} & \multicolumn{3}{|c|}{$\begin{array}{c}\text { Dry weight of plant at } \\
\text { harvest (g) }\end{array}$} & \multicolumn{3}{|c|}{ No. of fruits per plant } \\
\hline & & $\begin{array}{c}\text { I } \\
\text { year }\end{array}$ & $\begin{array}{c}\text { II } \\
\text { year }\end{array}$ & $\begin{array}{c}\text { Poole } \\
\text { d }\end{array}$ & $\begin{array}{c}\text { I } \\
\text { year }\end{array}$ & $\begin{array}{c}\text { II } \\
\text { year }\end{array}$ & Pooled & $\begin{array}{c}\text { I } \\
\text { year }\end{array}$ & $\begin{array}{c}\text { II } \\
\text { year }\end{array}$ & Pooled & $\begin{array}{c}\text { I } \\
\text { year }\end{array}$ & $\begin{array}{c}\text { II } \\
\text { year }\end{array}$ & $\begin{array}{c}\text { Poole } \\
\text { d }\end{array}$ \\
\hline $\mathbf{T}_{1}$ & Alachlor (PE)-1.5 kg ai/ha & 73.00 & 72.00 & 72.50 & 11.46 & 12.55 & 12.00 & 73.94 & 76.71 & 75.32 & 84.74 & 85.99 & 85.36 \\
\hline $\mathbf{T}_{2}$ & Alachlor (PE)-1.0 kg ai/ha & 69.00 & 67.50 & 68.25 & 9.71 & 10.80 & 10.25 & 70.49 & 73.26 & 71.87 & 80.62 & 82.00 & 81.31 \\
\hline $\mathbf{T}_{3}$ & $\begin{array}{l}\text { Alachlor (PE) }-1.5 \mathrm{~kg} \text { ai/ha }+1 \\
\mathrm{HW} \text { at } 45 \mathrm{DAT}\end{array}$ & 89.00 & 90.00 & 89.50 & 17.96 & 19.05 & 18.50 & 88.39 & 91.32 & 89.85 & 94.45 & 95.85 & 95.15 \\
\hline $\mathbf{T}_{4}$ & $\begin{array}{l}\text { Alachlor }(\mathrm{PE})-1.5 \mathrm{~kg} \text { ai } / \mathrm{ha}+2 \\
\mathrm{HW} \text { at } 45 \text { and } 60 \mathrm{DAT}\end{array}$ & 99.00 & 100.00 & 99.50 & 23.74 & 25.14 & 24.44 & $\begin{array}{c}104.8 \\
3\end{array}$ & $\begin{array}{c}107.6 \\
5\end{array}$ & 106.24 & 118.67 & $\begin{array}{c}120.0 \\
1\end{array}$ & $\begin{array}{c}119.3 \\
4\end{array}$ \\
\hline $\mathbf{T}_{5}$ & $\begin{array}{l}\text { Alachlor (PE) }-1.0 \mathrm{~kg} \text { ai/ha }+1 \\
\mathrm{HW} \text { at } 45 \mathrm{DAT}\end{array}$ & 82.00 & 82.50 & 82.25 & 15.15 & 16.20 & 15.67 & 84.61 & 87.64 & 86.12 & 92.40 & 93.94 & 93.17 \\
\hline $\mathbf{T}_{6}$ & $\begin{array}{l}\text { Alachlor }(\mathrm{PE})-1.0 \mathrm{~kg} \text { ai } / \mathrm{ha}+2 \\
\mathrm{HW} \text { at } 45 \text { and } 60 \mathrm{DAT}\end{array}$ & 95.00 & 96.00 & 95.50 & 19.34 & 20.44 & 19.89 & 97.07 & 99.84 & 98.45 & 102.33 & $\begin{array}{c}104.0 \\
0\end{array}$ & $\begin{array}{c}103.1 \\
6\end{array}$ \\
\hline $\mathbf{T}_{7}$ & Pendimethalin (PE)-1.5 kg ai/ha & 71.00 & 69.50 & 70.25 & 10.46 & 11.66 & 11.06 & 73.47 & 76.47 & 74.97 & 83.31 & 84.67 & 83.99 \\
\hline $\mathbf{T}_{8}$ & Pendimethalin (PE)-1.0 kg ai/ha & 64.00 & 62.50 & 63.25 & 8.36 & 9.56 & 8.96 & 64.47 & 67.47 & 65.97 & 78.62 & 79.95 & 79.28 \\
\hline $\mathbf{T}_{9}$ & $\begin{array}{l}\text { Pendimethalin (PE)-1.5 kg ai/ha+ } \\
1 \mathrm{HW} \text { at } 45 \mathrm{DAT}\end{array}$ & 79.00 & 78.00 & 78.50 & 14.18 & 15.28 & 14.73 & 82.35 & 85.35 & 83.85 & 89.83 & 90.84 & 90.33 \\
\hline $\mathbf{T}_{10}$ & $\begin{array}{l}\text { Pendimethalin (PE) }-1.5 \mathrm{~kg} \text { ai } / \mathrm{ha} \\
+2 \mathrm{HW} \text { at } 45 \text { and } 60 \mathrm{DAT}\end{array}$ & 97.00 & 98.00 & 97.50 & 20.82 & 21.87 & 21.34 & $\begin{array}{c}100.5 \\
0\end{array}$ & $\begin{array}{c}103.1 \\
2\end{array}$ & 101.81 & 103.52 & $\begin{array}{c}104.8 \\
1\end{array}$ & $\begin{array}{c}104.1 \\
6\end{array}$ \\
\hline $\mathbf{T}_{11}$ & $\begin{array}{l}\text { Pendimethalin (PE)-1.0 kg ai/ha } \\
+1 \mathrm{HW} \text { at } 45 \mathrm{DAT}\end{array}$ & 74.00 & 73.00 & 73.50 & 12.62 & 13.82 & 13.22 & 80.37 & 83.37 & 81.87 & 88.94 & 90.46 & 89.70 \\
\hline $\mathbf{T}_{12}$ & $\begin{array}{l}\text { Pendimethalin (PE) }-1.0 \mathrm{~kg} \text { ai/ha } \\
+2 \mathrm{HW} \text { at } 45 \text { and } 60 \mathrm{DAT}\end{array}$ & 93.00 & 93.50 & 93.25 & 18.57 & 19.72 & 19.15 & 94.78 & 97.56 & 96.17 & 99.56 & $\begin{array}{c}101.4 \\
5\end{array}$ & $\begin{array}{c}100.5 \\
0\end{array}$ \\
\hline $\mathbf{T}_{13}$ & Weed free check & 100.00 & 101.00 & $\begin{array}{c}100.5 \\
0\end{array}$ & 26.17 & 27.52 & 26.84 & $\begin{array}{c}110.9 \\
9\end{array}$ & $\begin{array}{c}114.0 \\
4\end{array}$ & 112.52 & 124.19 & $\begin{array}{c}125.4 \\
3\end{array}$ & $\begin{array}{c}124.8 \\
1\end{array}$ \\
\hline $\mathbf{T}_{14}$ & Unweeded check & 62.00 & 60.50 & 61.25 & 8.04 & 9.09 & 8.57 & 40.71 & 41.86 & 41.28 & 48.24 & 49.73 & 48.98 \\
\hline & Mean & 81.93 & 81.71 & 81.82 & 15.47 & 16.62 & 16.04 & 83.35 & 86.12 & 84.73 & 92.10 & 93.51 & 92.80 \\
\hline & S. Em $₫$ & 4.20 & 4.05 & 4.12 & 0.81 & 1.44 & 1.02 & 3.05 & 5.79 & 4.29 & 3.34 & 5.71 & 4.31 \\
\hline & C.D. @ 5\% & 12.82 & 12.37 & 12.59 & 2.46 & 4.41 & 3.13 & 9.30 & 17.70 & 13.11 & 10.21 & 17.44 & 13.18 \\
\hline
\end{tabular}


Table.4 Effect of weed control treatments on yield and yield attributes in chilli

\begin{tabular}{|c|c|c|c|c|c|c|c|c|c|c|}
\hline \multirow[t]{2}{*}{ Treatments } & \multirow[t]{2}{*}{ Treatment details } & \multicolumn{3}{|c|}{$\begin{array}{c}\text { Fruit weight per plant } \\
\text { (kg) }\end{array}$} & \multicolumn{3}{|c|}{ Yield per plot (kg) } & \multicolumn{3}{|c|}{ Yield per ha (q) } \\
\hline & & $\begin{array}{c}\text { I } \\
\text { year }\end{array}$ & $\begin{array}{c}\text { II } \\
\text { year }\end{array}$ & Pooled & $\begin{array}{c}\text { I } \\
\text { year }\end{array}$ & $\begin{array}{c}\text { II } \\
\text { year }\end{array}$ & Pooled & $\begin{array}{c}\text { I } \\
\text { year }\end{array}$ & $\begin{array}{c}\text { II } \\
\text { year }\end{array}$ & Pooled \\
\hline $\mathbf{T}_{1}$ & Alachlor (PE)-1.5 kg ai/ha & 0.94 & 0.98 & 0.96 & 35.80 & 37.32 & 36.56 & 220.32 & 230.39 & 225.70 \\
\hline $\mathbf{T}_{2}$ & Alachlor (PE)-1.0 kg ai/ha & 0.92 & 0.96 & 0.94 & 34.88 & 36.40 & 35.64 & 214.64 & 224.68 & 219.99 \\
\hline $\mathbf{T}_{3}$ & Alachlor (PE)-1.5 kg ai $/ \mathrm{ha}+1 \mathrm{HW}$ at $45 \mathrm{DAT}$ & 0.99 & 1.03 & 1.01 & 37.48 & 39.00 & 38.24 & 230.65 & 240.74 & 236.05 \\
\hline $\mathbf{T}_{4}$ & $\begin{array}{l}\text { Alachlor (PE)-1.5 kg ai/ha }+2 \mathrm{HW} \text { at } 45 \text { and } \\
60 \text { DAT }\end{array}$ & 1.08 & 1.12 & 1.10 & 40.86 & 42.38 & 41.62 & 251.46 & 261.62 & 256.93 \\
\hline $\mathbf{T}_{\mathbf{5}}$ & Alachlor (PE)-1.0 kg ai $/ \mathrm{ha}+1 \mathrm{HW}$ at $45 \mathrm{DAT}$ & 0.98 & 1.02 & 1.00 & 37.32 & 38.84 & 38.08 & 229.67 & 239.76 & 235.07 \\
\hline $\mathbf{T}_{6}$ & $\begin{array}{l}\text { Alachlor (PE)-1.0 kg ai/ha }+2 \mathrm{HW} \text { at } 45 \text { and } \\
60 \mathrm{DAT}\end{array}$ & 1.01 & 1.05 & 1.03 & 38.41 & 39.93 & 39.17 & 236.37 & 246.48 & 241.79 \\
\hline $\mathbf{T}_{7}$ & Pendimethalin (PE)-1.5 kg ai/ha & 0.93 & 0.97 & 0.95 & 35.35 & 36.87 & 36.11 & 217.56 & 227.61 & 222.92 \\
\hline $\mathbf{T}_{8}$ & Pendimethalin (PE)-1.0 kg ai/ha & 0.90 & 0.94 & 0.92 & 34.20 & 35.72 & 34.96 & 210.46 & 220.49 & 215.80 \\
\hline $\mathbf{T}_{9}$ & $\begin{array}{l}\text { Pendimethalin (PE)-1.5 kg ai/ha+ } 1 \mathrm{HW} \text { at } 45 \\
\text { DAT }\end{array}$ & 0.97 & 1.01 & 0.99 & 36.79 & 38.31 & 37.55 & 226.39 & 236.47 & 231.78 \\
\hline $\mathbf{T}_{10}$ & $\begin{array}{l}\text { Pendimethalin (PE) }-1.5 \mathrm{~kg} \text { ai } / \mathrm{ha}+2 \mathrm{HW} \text { at } 45 \\
\text { and } 60 \mathrm{DAT}\end{array}$ & 1.03 & 1.07 & 1.05 & 39.16 & 40.68 & 39.92 & 240.96 & 251.08 & 246.39 \\
\hline $\mathbf{T}_{11}$ & $\begin{array}{l}\text { Pendimethalin (PE)-1.0 kg ai/ha }+1 \mathrm{HW} \text { at } 45 \\
\text { DAT }\end{array}$ & 0.95 & 0.99 & 0.97 & 36.23 & 37.75 & 36.99 & 222.94 & 233.01 & 228.32 \\
\hline $\mathbf{T}_{12}$ & $\begin{array}{l}\text { Pendimethalin (PE)-1.0 kg ai/ha }+2 \mathrm{HW} \text { at } 45 \\
\text { and } 60 \text { DAT }\end{array}$ & 1.00 & 1.04 & 1.02 & 37.87 & 39.39 & 38.63 & 233.04 & 243.14 & 238.45 \\
\hline $\mathbf{T}_{13}$ & Weed free check & 1.11 & 1.16 & 1.14 & 42.21 & 44.11 & 43.16 & 259.75 & 272.28 & 266.42 \\
\hline $\mathbf{T}_{14}$ & Unweeded check & 0.41 & 0.43 & 0.45 & 15.39 & 16.15 & 16.91 & 94.71 & 99.69 & 104.38 \\
\hline & Mean & 0.94 & 0.98 & 0.97 & 35.89 & 37.35 & 36.68 & 220.64 & 230.53 & 226.43 \\
\hline & S. $\operatorname{Em} \pm$ & 0.95 & 0.04 & 0.04 & 1.32 & 1.66 & 1.36 & 8.15 & 10.23 & 8.38 \\
\hline & C.D. @ 5\% & 2.81 & 0.29 & 0.15 & 3.64 & 4.96 & 4.78 & 24.77 & 31.25 & 25.85 \\
\hline
\end{tabular}


Table.5 Effect of weed control treatments on growth parameters in garlic

\begin{tabular}{|c|c|c|c|c|c|c|c|}
\hline \multirow[t]{2}{*}{ Treatments } & \multirow[t]{2}{*}{ Treatment details } & \multicolumn{3}{|c|}{$\begin{array}{l}\text { Plant height at } 90 \\
\text { DAT }(\mathrm{cm})\end{array}$} & \multicolumn{3}{|c|}{$\begin{array}{l}\text { No. of leaves per plant at } \\
90 \text { DAT }\end{array}$} \\
\hline & & $\begin{array}{c}\text { I } \\
\text { year }\end{array}$ & $\begin{array}{c}\text { II } \\
\text { year }\end{array}$ & $\begin{array}{l}\text { Poole } \\
\text { d }\end{array}$ & $\begin{array}{c}\text { I } \\
\text { year }\end{array}$ & $\begin{array}{c}\text { II } \\
\text { year }\end{array}$ & Pooled \\
\hline $\mathbf{T}_{1}$ & Alachlor (PE)-1.5 kg ai/ha & 32.15 & 32.35 & 32.25 & 9.10 & 9.30 & 9.20 \\
\hline $\mathbf{T}_{2}$ & Alachlor (PE)-1.0 kg ai/ha & 30.00 & 29.85 & 29.93 & 8.45 & 8.65 & 8.55 \\
\hline $\mathbf{T}_{\mathbf{3}}$ & Alachlor (PE)-1.5 kg ai/ha $+1 \mathrm{HW}$ at $45 \mathrm{DAT}$ & 37.00 & 36.95 & 36.98 & 10.35 & 10.55 & 10.45 \\
\hline $\mathbf{T}_{4}$ & $\begin{array}{l}\text { Alachlor (PE)-1.5 kg ai } / \mathrm{ha}+2 \mathrm{HW} \text { at } 45 \text { and } \\
60 \mathrm{DAT}\end{array}$ & 42.40 & 41.55 & 41.98 & 12.00 & 12.20 & 12.10 \\
\hline $\mathbf{T}_{5}$ & Alachlor (PE)-1.0 kg ai $/ \mathrm{ha}+1 \mathrm{HW}$ at $45 \mathrm{DAT}$ & 36.65 & 35.85 & 36.25 & 10.05 & 10.35 & 10.20 \\
\hline $\mathbf{T}_{6}$ & $\begin{array}{l}\text { Alachlor (PE)-1.0 kg ai } / \mathrm{ha}+2 \mathrm{HW} \text { at } 45 \text { and } \\
60 \mathrm{DAT}\end{array}$ & 39.75 & 39.50 & 39.63 & 11.45 & 11.15 & 11.05 \\
\hline $\mathbf{T}_{7}$ & Pendimethalin (PE)-1.5 kg ai/ha & 31.05 & 31.00 & 31.03 & 8.80 & 9.00 & 8.90 \\
\hline $\mathbf{T}_{8}$ & Pendimethalin (PE)-1.0 kg ai/ha & 28.25 & 28.50 & 28.38 & 8.05 & 8.25 & 8.15 \\
\hline $\mathbf{T}_{9}$ & $\begin{array}{l}\text { Pendimethalin (PE)-1.5 kg ai/ha+ } 1 \mathrm{HW} \text { at } 45 \\
\text { DAT }\end{array}$ & 35.25 & 35.45 & 35.35 & 9.70 & 9.90 & 9.80 \\
\hline $\mathbf{T}_{10}$ & $\begin{array}{l}\text { Pendimethalin (PE)-1.5 kg ai/ha }+2 \mathrm{HW} \text { at } 45 \\
\text { and } 60 \mathrm{DAT}\end{array}$ & 41.65 & 40.75 & 41.20 & 11.25 & 12.00 & 11.85 \\
\hline $\mathbf{T}_{11}$ & $\begin{array}{l}\text { Pendimethalin (PE)-1.0 kg ai/ha }+1 \mathrm{HW} \text { at } 45 \\
\text { DAT }\end{array}$ & 35.00 & 34.30 & 34.65 & 9.40 & 9.60 & 9.50 \\
\hline $\mathbf{T}_{12}$ & $\begin{array}{l}\text { Pendimethalin (PE)-1.0 kg ai/ha }+2 \mathrm{HW} \text { at } 45 \\
\text { and } 60 \text { DAT }\end{array}$ & 37.70 & 38.50 & 38.10 & 10.60 & 10.80 & 10.70 \\
\hline $\mathbf{T}_{13}$ & Weed free check & 43.00 & 44.50 & 44.25 & 12.40 & 12.70 & 12.60 \\
\hline $\mathbf{T}_{14}$ & Unweeded check & 19.00 & 18.50 & 18.75 & 7.00 & 6.70 & 7.10 \\
\hline & Mean & 34.92 & 34.83 & 34.91 & 9.90 & 10.08 & 10.01 \\
\hline & S. $\mathbf{E m} \pm$ & 1.28 & 1.25 & 1.28 & 0.36 & 0.38 & 0.36 \\
\hline & C.D. @ 5\% & 3.92 & 3.81 & 3.92 & 1.11 & 1.15 & 1.10 \\
\hline
\end{tabular}


Table.6 Effect of weed control treatments on yield and yield attributes in garlic

\begin{tabular}{|c|c|c|c|c|c|c|c|c|c|c|c|c|c|}
\hline \multirow[t]{2}{*}{ Treatments } & \multirow[t]{2}{*}{ Treatment details } & \multicolumn{3}{|c|}{$\begin{array}{c}\text { Dry weight of plant at } \\
\text { 90 DAT (g) }\end{array}$} & \multicolumn{3}{|c|}{ Yield per plant (g) } & \multicolumn{3}{|c|}{ Yield per plot (kg) } & \multicolumn{3}{|c|}{ Yield per ha $(q)$} \\
\hline & & $\begin{array}{c}\text { I } \\
\text { year }\end{array}$ & $\begin{array}{c}\text { II } \\
\text { year }\end{array}$ & Pooled & $\begin{array}{c}\text { I } \\
\text { year }\end{array}$ & $\begin{array}{c}\text { II } \\
\text { year }\end{array}$ & Pooled & $\begin{array}{c}\text { I } \\
\text { year }\end{array}$ & $\begin{array}{c}\text { II } \\
\text { year }\end{array}$ & $\begin{array}{c}\text { Poole } \\
\text { d }\end{array}$ & $\begin{array}{c}\text { I } \\
\text { year }\end{array}$ & $\begin{array}{c}\text { II } \\
\text { year }\end{array}$ & $\begin{array}{c}\text { Poole } \\
\text { d }\end{array}$ \\
\hline $\mathbf{T}_{1}$ & Alachlor (PE)-1.5 kg ai/ha & 5.22 & 4.37 & 4.79 & 8.84 & 8.94 & 8.95 & 3.69 & 3.67 & 3.68 & 23.00 & 22.00 & 22.50 \\
\hline $\mathbf{T}_{2}$ & Alachlor (PE)-1.0 kg ai/ha & 4.32 & 3.21 & 3.77 & 8.47 & 8.80 & 8.64 & 3.31 & 3.32 & 3.31 & 20.40 & 20.45 & 20.65 \\
\hline $\mathbf{T}_{3}$ & $\begin{array}{l}\text { Alachlor (PE)- } 1.5 \mathrm{~kg} \text { ai } / \mathrm{ha}+1 \mathrm{HW} \\
\text { at } 45 \mathrm{DAT}\end{array}$ & 5.62 & 4.72 & 5.17 & 9.06 & 9.19 & 9.11 & 4.36 & 4.38 & 4.37 & 27.00 & 27.50 & 27.25 \\
\hline $\mathbf{T}_{4}$ & $\begin{array}{l}\text { Alachlor (PE)-1.5 kg ai } / \mathrm{ha}+2 \mathrm{HW} \\
\text { at } 45 \text { and } 60 \mathrm{DAT}\end{array}$ & 6.07 & 5.34 & 5.71 & 9.47 & 9.89 & 9.59 & 4.78 & 5.28 & 5.03 & 29.50 & 34.05 & 31.80 \\
\hline $\mathbf{T}_{5}$ & $\begin{array}{l}\text { Alachlor (PE)-1.0 kg ai } / \mathrm{ha}+1 \mathrm{HW} \\
\text { at } 45 \text { DAT }\end{array}$ & 5.53 & 4.67 & 5.10 & 9.00 & 9.15 & 9.08 & 4.28 & 4.32 & 4.30 & 26.50 & 27.00 & 26.75 \\
\hline $\mathbf{T}_{6}$ & $\begin{array}{l}\text { Alachlor (PE)-1.0 kg ai } / \mathrm{ha}+2 \mathrm{HW} \\
\text { at } 45 \text { and } 60 \mathrm{DAT}\end{array}$ & 5.85 & 5.16 & 5.50 & 9.23 & 9.44 & 9.23 & 4.61 & 4.71 & 4.66 & 28.45 & 29.00 & 28.70 \\
\hline $\mathbf{T}_{7}$ & Pendimethalin (PE)-1.5 kg ai/ha & 4.96 & 3.31 & 4.13 & 8.60 & 8.82 & 8.89 & 3.50 & 3.46 & 3.48 & 21.90 & 22.40 & 22.15 \\
\hline $\mathbf{T}_{8}$ & Pendimethalin (PE)-1.0 kg ai/ha & 4.22 & 3.10 & 3.66 & 8.18 & 8.15 & 8.16 & 3.12 & 3.10 & 3.11 & 19.10 & 19.05 & 19.05 \\
\hline $\mathbf{T}_{9}$ & $\begin{array}{l}\text { Pendimethalin (PE)-1.5 kg ai/ha+ } 1 \\
\mathrm{HW} \text { at } 45 \text { DAT }\end{array}$ & 5.41 & 4.56 & 4.98 & 8.97 & 9.07 & 9.02 & 4.00 & 4.01 & 4.00 & 24.80 & 25.40 & 25.10 \\
\hline $\mathbf{T}_{10}$ & $\begin{array}{l}\text { Pendimethalin (PE) }-1.5 \mathrm{~kg} \text { ai } / \mathrm{ha}+2 \\
\mathrm{HW} \text { at } 45 \text { and } 60 \mathrm{DAT}\end{array}$ & 5.96 & 5.30 & 5.63 & 9.29 & 9.47 & 9.45 & 4.68 & 4.79 & 4.74 & 28.90 & 31.00 & 29.95 \\
\hline $\mathbf{T}_{11}$ & $\begin{array}{l}\text { Pendimethalin }(\mathrm{PE})-1.0 \mathrm{~kg} \text { ai } / \mathrm{ha}+1 \\
\mathrm{HW} \text { at } 45 \mathrm{DAT}\end{array}$ & 5.32 & 4.46 & 4.89 & 8.95 & 9.05 & 9.00 & 3.88 & 3.86 & 3.87 & 24.00 & 24.50 & 24.25 \\
\hline $\mathbf{T}_{12}$ & $\begin{array}{l}\text { Pendimethalin }(\mathrm{PE})-1.0 \mathrm{~kg} \text { ai } / \mathrm{ha}+2 \\
\mathrm{HW} \text { at } 45 \text { and } 60 \mathrm{DAT}\end{array}$ & 5.70 & 4.79 & 5.24 & 9.21 & 9.29 & 9.15 & 4.46 & 4.48 & 4.47 & 27.60 & 28.30 & 27.95 \\
\hline $\mathbf{T}_{13}$ & Weed free check & 7.00 & 5.70 & 6.54 & 11.18 & 11.99 & 11.59 & 4.88 & 5.39 & 5.13 & 30.10 & 37.00 & 33.65 \\
\hline$T_{14}$ & Unweeded check & 2.85 & 2.93 & 2.89 & 3.66 & 4.36 & 3.88 & 2.28 & 2.09 & 2.19 & 14.11 & 14.10 & 14.09 \\
\hline & Mean & 5.29 & 4.40 & 4.86 & 8.72 & 8.97 & 8.84 & 3.98 & 4.06 & 4.02 & 24.67 & 25.84 & 25.28 \\
\hline & S. Em \pm & 0.41 & 0.43 & 0.21 & 0.40 & 0.39 & 0.34 & 0.19 & 0.26 & 0.21 & 1.16 & 2.45 & 1.70 \\
\hline & C.D. @ 5\% & 1.25 & 1.32 & 0.64 & 1.29 & 1.67 & 1.40 & 0.58 & 0.81 & 0.65 & 4.94 & 7.43 & 5.22 \\
\hline
\end{tabular}


Table.7 Effect of weed control treatments on economics in chilli +garlic intercropping

\begin{tabular}{|c|c|c|c|c|c|}
\hline Treatments & Treatment details & $\begin{array}{l}\text { Gross } \\
\text { returns (Rs) }\end{array}$ & $\begin{array}{l}\text { Cost of } \\
\text { cultivation (Rs) }\end{array}$ & $\begin{array}{l}\text { Net returns } \\
(\mathrm{Rs})\end{array}$ & $\begin{array}{l}\text { B:C } \\
\text { ratio }\end{array}$ \\
\hline $\mathbf{T}_{1}$ & Alachlor (PE)-1.5 kg ai/ha & $1,57,850$ & 79,500 & 78,350 & 1.98 \\
\hline $\mathbf{T}_{2}$ & Alachlor (PE)-1.0 kg ai/ha & $1,51,295$ & 79,200 & 72,095 & 1.91 \\
\hline $\mathbf{T}_{\mathbf{3}}$ & Alachlor (PE)-1.5 kg ai/ha $+1 \mathrm{HW}$ at $45 \mathrm{DAT}$ & $1,72,525$ & 81,500 & 91,025 & 2.11 \\
\hline $\mathbf{T}_{4}$ & $\begin{array}{l}\text { Alachlor (PE)- } 1.5 \mathrm{~kg} \text { ai/ha }+2 \mathrm{HW} \text { at } 45 \text { and } 60 \\
\text { DAT }\end{array}$ & $1,92,065$ & 83,500 & $1,08,565$ & 2.30 \\
\hline $\mathbf{T}_{5}$ & Alachlor (PE)-1.0 kg ai/ha $+1 \mathrm{HW}$ at $45 \mathrm{DAT}$ & $1,71,035$ & 81,200 & 89,835 & 2.10 \\
\hline $\mathbf{T}_{6}$ & $\begin{array}{l}\text { Alachlor (PE)-1.0 kg ai/ha }+2 \mathrm{HW} \text { at } 45 \text { and } 60 \\
\text { DAT }\end{array}$ & $1,78,295$ & 83,200 & 95,095 & 2.14 \\
\hline $\mathbf{T}_{7}$ & Pendimethalin (PE)-1.5 kg ai/ha & $1,55,760$ & 79,276 & 76,484 & 1.96 \\
\hline $\mathbf{T}_{8}$ & Pendimethalin (PE)-1.0 kg ai/ha & $1,46,000$ & 79,050 & 66,950 & 1.84 \\
\hline $\mathbf{T}_{9}$ & Pendimethalin (PE)-1.5 kg ai/ha+ $1 \mathrm{HW}$ at $45 \mathrm{DAT}$ & $1,66,090$ & 81,275 & 84,815 & 2.04 \\
\hline $\mathbf{T}_{10}$ & $\begin{array}{l}\text { Pendimethalin (PE)-1.5 kg ai/ha }+2 \mathrm{HW} \text { at } 45 \text { and } \\
60 \text { DAT }\end{array}$ & $1,83,095$ & 83,275 & 99,820 & 2.19 \\
\hline $\mathbf{T}_{11}$ & $\begin{array}{l}\text { Pendimethalin (PE)-1.0 kg ai/ha }+1 \mathrm{HW} \text { at } 45 \\
\text { DAT }\end{array}$ & $1,62,660$ & 81,050 & 81,610 & 2.00 \\
\hline $\mathbf{T}_{12}$ & $\begin{array}{l}\text { Pendimethalin (PE)-1.0 kg ai/ha }+2 \mathrm{HW} \text { at } 45 \text { and } \\
60 \text { DAT }\end{array}$ & $1,75,125$ & 83,050 & 92,075 & 2.10 \\
\hline $\mathbf{T}_{13}$ & Weed free check & $2,00,510$ & 92,600 & $1,07,910$ & 2.16 \\
\hline $\mathbf{T}_{14}$ & Unweeded check & 80,370 & 78,600 & 1,770 & 1.02 \\
\hline \multicolumn{2}{|r|}{ Mean } & - & - & - & - \\
\hline \multicolumn{2}{|r|}{ S. $\operatorname{Em} \pm$} & - & - & - & - \\
\hline \multicolumn{2}{|r|}{ C.D. @ 5\% } & - & - & - & - \\
\hline
\end{tabular}


Treatment $\mathrm{T}_{4}$ was found significant for highest plant height $(99.50 \mathrm{~cm})$, number of branches/ plant (24.44), dry weight of plant $(106.24 \mathrm{~g})$ and number of fruits/plant (119.34) in chilli (Table 3). The results showed that highest fruit weight /plant $(1.10 \mathrm{~kg})$ was recorded in $\mathrm{T}_{4}$ followed by $\mathrm{T}_{10}(1.05 \mathrm{~kg})$ and minimum was recorded from the unweeded check, $\mathrm{T}_{14}(0.45 \mathrm{~kg})$. Reduced yield from the unweeded plot may be attributed to increased competition for light, soil moisture and nutrients. Rajkumara(2009) found similar results on fruit weight of chilli against different control measures. Fruit yield per plot $(41.62 \mathrm{~kg})$ and yield per ha $(256.93 \mathrm{q})$ of green chilli was found highest in $\mathrm{T}_{4}$ while lowest was found in $\mathrm{T}_{14}(16.91 \mathrm{~kg}$ and 104.38 q, respectively) (Table - 4). Ningappa (2013) and Shil and Adhikary (2014) also reported similar findings in chilli.

Yield and yield components of garlic varied significantly among various weed control treatments. Treatment $\mathrm{T}_{4}$ was recorded significantly higher plant height $(41.98 \mathrm{~cm})$ and number of leaves/plant at harvest (12.10) in garlic (Table- 5). Weed free check recorded significantly highest dry weight of plant $(6.54 \mathrm{~g})$, yield per plant (11.59 g), yield per plot $(5.13 \mathrm{~kg})$ and yield per ha $(33.65 \mathrm{q})$. Among the chemical treatments, $\mathrm{T}_{4}$ recorded highest dry weight of plant $(5.71 \mathrm{~g})$, yield per plant $(9.59 \mathrm{~g})$, yield per plot $(5.03 \mathrm{~kg})$ and yield per ha (31.80q)followed by $\mathrm{T}_{10}$ (Table $6)$. The increase in plant dry matter and yield per plant in these treatments could be attributed to lower weed count and higher weed control efficiency which ultimately resulted in better crop growth leading to higher productivity. The similar results were quoted by Singh et al., (2002), Mohammad and Imran (2003) and Siddu et al., (2018) in garlic.

The economics of chilli +garlic intercropping indicated that weed free check recorded highest gross returns (Rs.2,00,510) followed by $\mathrm{T}_{4}$ (Rs.1,92,065). Cost of cultivation was highest in weed free check (Rs.92,600) followed by $\mathrm{T}_{4}$ (Rs.83,500). The higher cost of cultivation is due to increased labour charges incurred during hand weeding. Highest net returns (Rs.1,08,565) and benefit: cost ratio ( $\mathrm{B}: \mathrm{C}$ ratio) (2.30) was achieved by $\mathrm{T}_{4}$ followed by $\mathrm{T}_{10}$ (Table 7). The higher B:C ratio in $T_{4}$ is due to higher net returns and lower cost of cultivation in comparison with weed free check. These results are in line with the findings reported by Biradar (1999) and Singh et al., (2011).

In conclusion, pre-emergent application of alachlor@1.5 kg ai/ha + 2 HW at 45 and 60 DAT recorded the lowest pooled dry matter of weeds, population of monocot and dicot weeds and thus exhibited the highest weed control efficiency, highest yield per ha, net returns and $\mathrm{B}: \mathrm{C}$ ratio in chilli + garlic intercropping system. Thus it can be recommended as the best weed control treatment for chilli + garlic intercropping system.

\section{References}

Biradar, S., 1999, Integrated weed management in chilli under northern transitional tract of Karnataka. M.Sc. (Agri.) Thesis, Univ. Agric. Sci., Dharwad (India).

Chaudhari, D. D., Patel, V. J., Patel, H. K., Aakashmishra, Patel, B. D. and Parmar, D. J., 2017, Integrated control of complex weed flora in garlic. Res. on Crops 18(4): 668-674.

Gill, G. S. and Vijayakumar, 1969, Weed index- a new method for reporting weed control trials. Indian J. Agron., 16: $96-98$

Gomez, K. A and Gomez, A. A., 1984, Statistical procedures for agricultural research. $2^{\text {nd }}$ edition, Singapore, 
Johnwilley \& Sons.

Mohammad, K. and Imran, A, 2003, Effects of pre and post-emergence herbicides on weed control and garlic bulb yield. Sarhad J Agri., 19:105-111.

Mohite, K. K., Alekar, A. N., Murade, M.N. and Deshmukh, G.N., 2015, Influence of pre and post emergence herbicides on yield and quality of garlic (Allium sativum). J. Hort., 2(2):1-5.

Ningappa, 2013, Sequential application of pre and post emergent herbicides for weed management in chilli + onion + cotton intercropping system. M.Sc. (Agri.) Thesis, Univ. of Agril. Sciences, Dharwad (India).

Rajkumara, S., 2009. Weed management in onion -chilli cotton relay intercropping in rainfed vertisols. Ph.D. Thesis, University of Agricultural Sciences, Dharwad
(India).

Shil, S. and Adhikary, P., 2014, weed management in transplanted chilli. Indian J Weed Sci., 46(3): 261-263

Siddhu, G.M., Patil, B.T., Bachkar, C.B. and Handal, B.B., 2018, Weed management in garlic. $J$. Pharmacognosy Phytochemistry, 7(1): 1440-1444.

Singh R, Nandal, T.R. and Verma, S.G., 2002, Studies on weed management in garlic (Allium sativum L.). Indian $J$ Weed Sci., 34: 80-81.

Singh, U., Hiremath, S. M., Halikatti, S. I., Shashidhara, G. B. and Patil, P. L., 2011, Evaluation of herbicides for weed control in rainfed transplanted chilli (Capsicum annum L.). Karnataka J Agri. Sci., 24(2): 125128.

\section{How to cite this article:}

Vilas D. Gasti and Snehasish Chakravorty. 2019. Integrated Weed Management in Chilli + Garlic Intercropping System. Int.J.Curr.Microbiol.App.Sci. 8(01): 3100-3110. doi: https://doi.org/10.20546/ijcmas.2019.801.331 